\title{
On some products in pro-categories and shape categories
}

\section{Pop}

\begin{abstract}
This paper has two parts. In the first part we present a case in which we can calculate in a simple way the product of two inverse systems as object in a pro-category of a category with directed products, without counting systems at the same set of indices. In the second part, which is related to some papers of Keesling [4], Kodama [5], Mardešić [7], and Dydak-Mardešić [10], we give a sufficient condition by which a directed product of a category $\mathcal{T}$ is transformed by a shape functor $S: \mathcal{T} \rightarrow S h(\mathcal{T}, \mathcal{P})$ into a directed product. This theorem is applied to the toplogical shape category Sh(Top) where some examples are obtained.
\end{abstract}

\section{Introduction}

Shape theory is an extension of the homotopy theory from CW-complexes to arbitrary toplogical spaces. More precisely, let HTop denote the homotopy category, i.e., the category whose objects are topological spaces $X$ and whose morphisms are homotopy classes $[f]$ of continuous mappings $f: X \rightarrow Y$. Homotopy theory studies the restriction HPol of HTop to the class of spaces having the homotopy type of polyhedra. This class includes CW-complexes and ANR's for metric spaces. Shape theory studies the shape category Sh(Top), which is a modification of HTop. Its objects are all topological spaces. The morphisms are obtained by a process of approximation which uses the morphisms from HPol. Shape theory also studies the shape functor $S: H T o p \rightarrow$

Key Words: Products in a category, inverse systems, pro-category, shape category.

2010 Mathematics Subject Classification: Primary 55P55, 54C56, 18A30, 54B10, 54B35. Received: 29.10.2018

Accepted: 30.11.2018 
$S h(T o p)$. This functor keeps objects fixed, i.e., $S(X)=X$, and on HPol it is an isomorphism. Consequently, for spaces having the homotopy type of polyhedra, shape theory reduces to standard homotopy theory. It is generally considered that shape theory is the correct substitute for homotopy theory when one works with spaces beyond the HPol class (see [9]). The functors $H:$ Top $\rightarrow$ HTop, $S: H T o p \rightarrow S h(T o p)$ and the composition $S h:=S H: T o p \rightarrow S h(T o p)$ have a number of analogous properties. But there are also some exceptions. Thus, if for topological spaces $X$ and $Y, X \times Y$ denotes their Cartesian product with $\pi_{X}: X \times Y \rightarrow X$ and $\pi_{Y}: X \times Y \rightarrow Y$ the canonical projections, then it is well known that $\left(X \times Y, \operatorname{Sh}\left(\pi_{X}\right), \operatorname{Sh}\left(\pi_{Y}\right)\right)$ is not the product of $X$ and $Y$ in $\operatorname{Sh}(\mathrm{Top})$. In fact, James Keesling [4] has exhibited a noncompact subspace $X \subset \mathbb{R}^{2}$ for which $X \times X$ is not the product of $X$ with itself in Sh(Top). This example ( which is discussed in Section 4) has raised interest in finding pairs $(X, Y)$ of topological spaces for which $\left(X \times Y, S h\left(\pi_{X}\right), S h\left(\pi_{Y}\right)\right)$ is the product of $X$ and $Y$ in the shape category $\mathrm{Sh}$ (Top). Thus, in the same paper, Keesling proved that for compact Hausdorff spaces $X$ and $Y$, the answer is positive. Another special case when this statement is true is the case when both $X$ and $Y$ belong to the class HPol. This is because $X, Y \in \mathrm{HPol}$ imply $X \times Y \in \mathrm{HPol}$ and every shape morphism $F: Z \rightarrow X$ to a space $X \in \mathrm{HPol}$ admits a unique homotopy class $[f]=H(f)$ of a mapping $f: X \rightarrow Y$ so that $F=S[f]$. Then Sibe Mardešic proved in [7] that if a compact Haudorff space $X$ has the property that, for every polyhedron $P,\left(X \times P, S h\left(\pi_{X}\right), S h\left(\pi_{P}\right)\right)$ is a product in $\operatorname{Sh}(T o p)$, then $\left(X \times Y, S h\left(\pi_{X}\right), S h\left(\pi_{Y}\right)\right)$ is a product of $X$ and $Y$ in $\operatorname{Sh}(\mathrm{Top})$, for every topological space $Y$. Later, in [10] Jerzy Dydak and Sibe Mardešić found a metric continuum $X$ and a polyhedron $P$ such that the Cartesian product $X \times P$ fails to be the product of $X$ and $P$ in the shape category Sh(Top)(see Section 4). Finally, in the context of the same problem, Yuskihiro Kodama [5] proved that if $X$ is a compact and $Y$ is a paracompact space then $\operatorname{Sh}(X \times Y)$ is uniquely determined by $S h(X)$ and $S h(Y)$.

This paper has two parts. In the first part we present a case in which we can calculate in a simple way the product of two inverse systems as object in a pro-category of a category with directed products, without counting systems at the same set of indices. In the second part, which is related to some papers of Keesling [4], Kodama [5], Mardešić [7], and Dydak-Mardešić [10], we give a sufficient condition by which a directed product of a category $\mathcal{T}$ is transformed by a shape functor $S: \mathcal{T} \rightarrow S h(\mathcal{T}, \mathcal{P})$ into a directed product. This theorem is applied to the topological shape category $\mathrm{Sh}(\mathrm{Top})$ where some examples are obtained. 


\section{Some products in a pro-category}

Let $\mathcal{C}$ be a category with directed products, and $\mathbf{X}=\left(X_{\lambda}, p_{\lambda \lambda^{\prime}}, \Lambda\right), \mathbf{Y}=$ $\left(Y_{\mu}, q_{\mu \mu^{\prime}}, M\right)$ two inverse systems in $\mathcal{C}$. If $\mathcal{C}$ has products, then for two morphisms $f: X \rightarrow Y, f^{\prime}: X^{\prime} \rightarrow Y^{\prime}$ in the category $C$ there exists a unique morphism $f \times f^{\prime}: X \times X^{\prime} \rightarrow Y \times Y^{\prime}$ in $\mathcal{C}$, satisfying the relations

$$
\pi_{Y} \circ\left(f \times f^{\prime}\right)=f \circ \pi_{X}, \quad \pi_{Y^{\prime}} \circ\left(f \times f^{\prime}\right)=f^{\prime} \circ \pi_{X^{\prime}} .
$$

In addition, if $g: Y \rightarrow Z$ and $g^{\prime}: Y^{\prime} \rightarrow Z^{\prime}$ are two other morphisms in $\mathcal{C}$, then

$$
\left(g \times g^{\prime}\right) \circ\left(f \times f^{\prime}\right)=(g \circ f) \times\left(g^{\prime} \circ f^{\prime}\right) .
$$

Now consider the set $N=\Lambda \times M=\{\nu:=(\lambda, \mu) \mid \lambda \in \Lambda, \mu \in M\}$, with the order relation $\nu=(\lambda, \mu) \leq \nu^{\prime}=\left(\lambda^{\prime}, \mu^{\prime}\right)$ if and only if $\lambda \leq \lambda^{\prime}$ and $\mu \leq \mu^{\prime}$. Then $(N, \leq)$ is a directed set. On this set we index the following objects and morphisms of the category $\mathcal{C}: Z_{\nu}:=X_{\lambda} \times Y_{\mu}, r_{\nu \nu^{\prime}}:=p_{\lambda \lambda^{\prime}} \times q_{\mu \mu^{\prime}}$, for $\nu=(\lambda, \mu) \leq \nu^{\prime}=\left(\lambda^{\prime}, \mu^{\prime}\right)$. Then, using relations (2.1) and (2.2), we can easily prove that $\mathbf{Z}:=\left(Z_{\nu}, r_{\nu \nu^{\prime}}, N\right)$ is an inverse system in the category $\mathcal{C}$.

Theorem 2.1. Supose there exist two increasing functions $\varphi:(\Lambda, \leq) \rightarrow(M, \leq$ ) and $\psi:(M, \leq) \rightarrow(\Lambda, \leq)$, satisfying the following condition: for any pair $(\lambda, \mu) \in \Lambda \times M$, there exists either an index $\lambda^{\prime} \in \Lambda$, with $\lambda^{\prime} \geq \lambda, \varphi\left(\lambda^{\prime}\right) \geq \mu$, or an index $\mu^{\prime} \in M$, with $\mu^{\prime} \geq \mu, \psi\left(\mu^{\prime}\right) \geq \lambda$. Then for the above defined inverse system $\mathbf{Z}:=\left(Z_{\nu}, r_{\nu \nu^{\prime}}, N\right)$ there exist two pro-morphisms $\pi_{\mathbf{X}}: \mathbf{Z} \rightarrow \mathbf{X}, \pi_{\mathbf{Y}}:$ $\mathbf{Z} \rightarrow \mathbf{Y}$, such that the triplet $\left(\mathbf{Z}, \pi_{\mathbf{Z}}, \pi_{\mathbf{Y}}\right)$ is a directed product of $\mathbf{X}$ and $\mathbf{Y}$ in pro-e.

Proof. For $\pi_{\mathbf{X}}: \mathbf{Z}=\left(Z_{\nu}, r_{\nu \nu^{\prime}}, N\right) \rightarrow \mathbf{X}=\left(X_{\lambda}, p_{\lambda \lambda^{\prime}}, \Lambda\right)$, we put $\pi_{\mathbf{X}}=\left(\pi_{\lambda}, \bar{\varphi}\right)$, with $\bar{\varphi}: \Lambda \rightarrow N$ the function given by $\bar{\varphi}(\lambda)=(\lambda, \varphi(\lambda))$, and $\pi_{\lambda}: Z_{\bar{\varphi}(\lambda)} \rightarrow$ $X_{\lambda}$ the projection $\pi_{X_{\lambda}}$. If $\lambda^{\prime} \geq \lambda$, we have $\bar{\varphi}\left(\lambda^{\prime}\right) \geq \bar{\varphi}(\lambda)$, and by (2.1), $\pi_{\lambda} \circ r_{\bar{\varphi}(\lambda) \bar{\varphi}\left(\lambda^{\prime}\right)}=\pi_{X_{\lambda}} \circ\left(p_{\lambda \lambda^{\prime}} \times q_{\varphi(\lambda) \varphi\left(\lambda^{\prime}\right)}\right)=p_{\lambda \lambda^{\prime}} \circ \pi_{X_{\lambda^{\prime}}}=p_{\lambda \lambda^{\prime}} \circ \pi_{\lambda^{\prime}}$. Therefore $\pi_{\mathbf{X}}$ is a morphism of inverse systems. Analogously, we define $\pi_{\mathbf{Y}}=\left(\pi_{\mu}, \bar{\psi}\right)$, where $\bar{\psi}: M \rightarrow N$ is given by $\bar{\psi}(\mu)=(\psi(\mu), \mu)$, and $\pi_{\mu}: Z_{\bar{\psi}(\mu)} \rightarrow Y_{\mu}$, defined as $\pi_{Y_{\mu}}: X_{\psi(\mu)} \times Y_{\mu} \rightarrow Y_{\mu}$. Then if $\mu \leq \mu^{\prime}$, we have $\pi_{\mu} \circ r_{\bar{\psi}(\mu) \bar{\psi}\left(\mu^{\prime}\right)}=$ $\pi_{Y_{\mu}} \circ\left(p_{\psi(\mu) \psi\left(\mu^{\prime}\right)} \times q_{\mu \mu^{\prime}}\right)=q_{\mu \mu^{\prime}} \circ \pi_{Y_{\mu^{\prime}}}=q_{\mu \mu^{\prime}} \circ \pi_{\mu^{\prime}}$. Therefore $\pi_{Y}$ is also a morphism of inverse systems.

Now suppose that in category pro- $\mathcal{C}$ two arbitrary morphisms, represented by the morphisms of inverse systems $s_{\mathbf{X}}: \mathbf{S} \rightarrow \mathbf{X}$ and $s_{\mathbf{Y}}: \mathbf{S} \rightarrow \mathbf{Y}$, are given. We have to prove that there exists a unique morphism $s_{\mathbf{Z}}: \mathbf{S} \rightarrow \mathbf{Z}$ in pro $-\mathcal{C}$, so that $\pi_{\mathbf{X}} \circ s_{\mathbf{Z}}=s_{\mathbf{X}}$ and $\pi_{\mathbf{Y}} \circ s_{\mathbf{Z}}=s_{\mathbf{Y}}$. Suppose $\mathbf{S}=\left(S_{k}, \sigma_{k k^{\prime}}, K\right), s_{\mathbf{X}}=$ $\left(s_{\lambda}, \theta_{\mathbf{X}}\right), \theta_{\mathbf{X}}: \Lambda \rightarrow K, s_{\lambda}: S_{\theta_{\mathbf{X}}(\lambda)} \rightarrow X_{\lambda}$, and $s_{\mathbf{Y}}=\left(s_{\mu}, \theta_{\mathbf{Y}}\right), \theta_{\mathbf{Y}}: M \rightarrow K$, $s_{\mu}: S_{\theta_{\mathbf{Y}}(\mu)} \rightarrow Y_{\mu}$. Then we define an increasing function $\theta_{\mathbf{Z}}: \Lambda \times M \rightarrow K$ in 
the following way: if $(\lambda, \mu) \in \Lambda \times M$, consider $\theta_{\mathbf{Z}}((\lambda, \mu))=k \in K$ an index so that $\left.k \geq \theta_{\mathbf{X}}(\lambda), \theta_{\mathbf{Y}}(\mu)\right)$. By this we can consider $s_{\mathbf{Z}}=\left(s_{(\lambda, \mu)}, \theta_{\mathbf{Z}}\right)$, with $s_{(\lambda, \mu)}: S_{\theta_{\mathbf{Z}}((\lambda, \mu))} \rightarrow Z_{(\lambda, \mu)}=X_{\lambda} \times Y_{\mu}$, the morphism induced by the pair of morphisms in $\mathrm{C}$

$$
s_{\lambda} \circ \sigma_{\theta_{\mathbf{X}}(\lambda) k}: S_{k} \rightarrow X_{\lambda}, \quad s_{\mu} \circ \sigma_{\theta_{\mathbf{Y}}(\mu) k}: S_{k} \rightarrow Y_{\mu} .
$$

Therefore the morphism $s_{(\lambda, \mu)}$ satisfies the conditions

$$
\begin{aligned}
& \pi_{X_{\lambda}} \circ s_{(\lambda, \mu)}=s_{\lambda} \circ \sigma_{\theta_{\mathbf{X}}(\lambda) k}, \\
& \pi_{Y_{\mu}} \circ s_{(\lambda, \mu)}=s_{\mu} \circ \sigma_{\theta_{\mathbf{Y}}(\mu) k} .
\end{aligned}
$$

Now let us verify that $s_{\mathbf{Z}}=\left(s_{(\lambda, \mu)}, \theta_{\mathbf{Z}}\right)$ is a morphism of inverse systems. Suppose that $\left(\lambda^{\prime}, \mu^{\prime}\right) \geq(\lambda, \mu)$. Consider $\bar{k} \in K, k \geq k=\theta_{\mathbf{Z}}((\lambda, \mu)), k^{\prime}=$ $\theta_{\mathbf{Z}}\left(\left(\lambda^{\prime}, \mu^{\prime}\right)\right)$ so that

$$
p_{\lambda \lambda^{\prime}} \circ s_{\lambda^{\prime}} \circ \sigma_{\theta_{X}\left(\lambda^{\prime}\right) \bar{k}}=s_{\lambda} \circ \sigma_{\theta_{X}(\lambda) \bar{k}}
$$

which can be written as

$$
\left(p_{\lambda \lambda^{\prime}} \circ s_{\lambda^{\prime}} \circ \sigma_{\theta_{X}\left(\lambda^{\prime}\right) k^{\prime}}\right) \circ \sigma_{k^{\prime} \bar{k}}=s_{\lambda} \circ \sigma_{\theta_{X}(\lambda) k} \circ \sigma_{k \bar{k}} .
$$

This implies

$$
\pi_{X_{\lambda}} \circ s_{(\lambda, \mu)} \circ \sigma_{k \bar{k}}=\pi_{X_{\lambda}} \circ\left(p_{\lambda \lambda^{\prime}} \times q_{\mu \mu^{\prime}}\right) \circ s_{\left(\lambda^{\prime}, \mu^{\prime}\right)} \circ \sigma_{k^{\prime} \bar{k}} .
$$

Similarly we obtain

$$
\pi_{Y_{\mu}} \circ s_{(\lambda, \mu)} \circ \sigma_{k \bar{k}}=\pi_{Y_{\mu}} \circ\left(p_{\lambda \lambda^{\prime}} \times q_{\mu \mu^{\prime}}\right) \circ s_{\left(\lambda^{\prime}, \mu^{\prime}\right)} \circ \sigma_{k^{\prime} \bar{k}} \cdot \sigma_{k^{\prime} \bar{k}} .
$$

Relations (2.5) and (2.6) imply the relation

$$
s_{(\lambda, \mu)} \circ \sigma_{k \bar{k}}=\left(p_{\lambda \lambda^{\prime}} \times q_{\mu \mu^{\prime}}\right) \circ s_{\left(\lambda^{\prime}, \mu^{\prime}\right)} \circ \sigma_{k^{\prime} \bar{k}} .
$$

Therefore $s_{\mathbf{Z}}$ is a morphism of inverse systems and defines a morphism in pro $-\mathcal{C}$. In addition, by relations (2.3) and (2.4) we obtian

$$
\pi_{\mathbf{X}} \circ s_{\mathbf{Z}}=s_{\mathbf{X}}, \quad \pi_{\mathbf{Y}} \circ s_{\mathbf{Z}}=s_{\mathbf{Y}} .
$$

We now have to prove the uniqueness of the morphism $s_{\mathbf{Z}}$ in the category pro $-\mathcal{C}$. Let $s_{\mathbf{Z}}^{\prime}: \mathbf{S} \rightarrow \mathbf{Z}$ be an arbitary morphism in pro $-\mathcal{C}$ satisfying the conditions

$$
\pi_{\mathbf{X}} \circ s_{\mathbf{Z}}^{\prime}=s_{\mathbf{X}}, \quad \pi_{\mathbf{Y}} \circ s_{\mathbf{Z}}^{\prime}=s_{\mathbf{Y}} .
$$

Suppose that $s_{\mathbf{Z}}^{\prime}$ is given by a morphism of inverse systems, $\left(s_{(\lambda, \mu)}^{\prime}, \theta_{\mathbf{Z}}^{\prime}\right), \theta_{\mathbf{Z}}^{\prime}$ : $\Lambda \times M \rightarrow K$. We have to prove that $s_{\mathbf{Z}}^{\prime}=s_{\mathbf{Z}}$ in the category pro $-\mathcal{C}$. This 
means that for $(\lambda, \mu) \in \Lambda \times M$ there exists $\bar{k} \in K, \bar{k} \geq \theta_{\mathbf{Z}}((\lambda, \mu)), \theta_{\mathbf{Z}}^{\prime}((\lambda, \mu))$ so that

$$
s_{(\lambda, \mu)} \circ \sigma_{\theta_{\mathbf{Z}}((\lambda, \mu)) \bar{k}}=s_{(\lambda, \mu)}^{\prime} \circ \sigma_{\theta_{\mathbf{Z}}^{\prime}((\lambda, \mu)) \bar{k}} .
$$

By the first relation (2.8), we have that the morphisms $\pi_{\lambda} \circ s_{(\lambda, \varphi(\lambda))}^{\prime}$ : $S_{\theta_{\mathbf{z}}^{\prime}(\lambda, \varphi(\lambda))} \rightarrow X_{\lambda}$ and $s_{\lambda}: S_{\left.\left.\theta_{\mathbf{X}}\right) \lambda\right)} \rightarrow X_{\lambda}$ are equivalent. By this we obtain a relation

$$
\pi_{\lambda} \circ s_{(\lambda, \varphi(\lambda))}^{\prime} \circ \sigma_{\theta_{\mathbf{Z}}^{\prime}(\lambda, \varphi(\lambda)) k}=s_{\lambda} \circ \sigma_{\theta_{\mathbf{X}}(\lambda) k} .
$$

Similarly, the second relation (2.8) induces a realtion

$$
\pi_{\mu} \circ s_{(\psi(\mu), \mu)}^{\prime} \circ \sigma_{\theta_{\mathbf{Z}}^{\prime}(\psi(\mu), \mu) k}=s_{\mu} \circ \sigma_{\theta_{\mathbf{Y}}(\lambda) k} .
$$

By (2.10) and (2.11), we obtain the relation (2.9), for $(\lambda, \mu)=(\lambda, \varphi(\lambda))$ and $(\lambda, \mu)=(\psi(\mu), \mu)$. Then by the conditions imposed on the functions $\varphi$ and $\psi$, we conclude that $(2.9)$ is verified for all pairs $(\lambda, \mu)$, which implies $s_{\mathbf{Z}}^{\prime}=s_{\mathbf{Z}}$ in pro $-C$. And this finishes the proof.

Remark 2.2. If the pair of inverse systems $(\mathbf{X}, \mathbf{Y})$ satisfies the condition from Theorem 2.1, then as usual, the inverse system $\mathbf{Z}$ of the product $\mathbf{X} \times \mathbf{Y}$ does not depend, up to an isomorphism, on the pair of functions $(\varphi, \psi)$, or another way to get the product.

Example 2.3. 1) For each inverse system $\mathbf{X}=\left(X_{\lambda}, p_{\lambda \lambda^{\prime}}, \Lambda\right)$, the pair $(\mathbf{X}, \mathbf{X})$ satisfies the condition from Theorem 2.1 , with $\varphi=\psi=1_{\Lambda}$.

2) Any pair of inverse systems with the same set of indices satisfies the condition from Theorem 2.1. Particularly, this is the case of a pair of inverse sequences.

3) Any pair consisting of an arbitary inverse system and a rudimentary system satisfies the condition from Theorem 2.1.

4) If $\mathbf{X}=\left(X_{\lambda}, p_{\lambda \lambda^{\prime}}, \Lambda\right), \mathbf{Y}=\left(Y_{\mu}, q_{\mu \mu^{\prime}}, M\right)$, in a category $\mathcal{C}$ with products, and if $(\Lambda, \leq)$ is a cofinal subset of $(M, \leq)$ such that there exists an increasing function $\psi:(M, \leq) \rightarrow(\Lambda, \leq)$, then taking the inclusion $(\Lambda, \leq) \hookrightarrow(M, \leq)$ as function $\varphi$, the condition from Theorem 2.1 is satisfied: for $(\lambda, \mu) \in \Lambda \times M$, there exists $\lambda^{\prime} \in \Lambda, \lambda^{\prime} \geq \lambda$ and $\varphi\left(\lambda^{\prime}\right)=\lambda^{\prime} \geq \mu$.

5) If we have a pair $(\mathbf{X}, \mathbf{Y})$ which satisfies the condition from Theorem 2.1, and some pro-isomorphisms $\mathbf{f}: \mathbf{X} \rightarrow \mathbf{X}^{\prime}, \mathbf{g}: \mathbf{Y} \rightarrow \mathbf{Y}^{\prime}$, then $\left(\mathbf{X} \times \mathbf{Y}, \mathbf{f} \circ \pi_{\mathbf{X}}, \mathbf{g} \circ\right.$ $\left.\pi_{\mathbf{Y}}\right)$ is a product of $\mathbf{X}^{\prime}$ and $\mathbf{Y}^{\prime}$ as pro-objects.

Corollary 2.4. Let $\mathcal{C}$ be a category with products, and $\mathbf{f}=\left[\left(f_{\mu}, \phi\right)\right]: \mathbf{X} \rightarrow$ $\mathbf{Y}=\left(Y_{\mu}, q_{\mu \mu^{\prime}}, M\right), \overline{\mathbf{f}}=\left[\left(\bar{f}_{\bar{\mu}}, \bar{\phi}\right)\right]: \overline{\mathbf{X}} \rightarrow \overline{\mathbf{Y}}=\left(\bar{Y}_{\bar{\mu}}, \bar{q}_{\overline{\mu \mu^{\prime}}}, M\right)$ be morphisms in pro - $\mathcal{C}$. If $(\mathbf{X}, \overline{\mathbf{X}})$ and $(\mathbf{Y}, \overline{\mathbf{Y}})$ satisfy the condition from Theorem 2.1, then the pro-morphism $\mathbf{f} \times \overline{\mathbf{f}}: \mathbf{X} \times \overline{\mathbf{X}} \rightarrow \mathbf{Y} \times \overline{\mathbf{Y}}$ can be given by the morphism of inverse systems $\left(f_{\mu} \times \bar{f}_{\bar{\mu}}, \phi \times \bar{\phi}\right)$. 
Remark 2.5. Suppose that $\mathbf{X}=\left(X_{\lambda}, p_{\lambda \lambda^{\prime}}, \Lambda\right)$ and $\mathbf{Y}=\left(Y_{\mu}, q_{\mu \mu^{\prime}}, M\right)$ are two inverse systems for each there exist some functions $\varphi:(\Lambda, \leq) \rightarrow(M, \leq)$ and $\psi:(M, \leq) \rightarrow(\Lambda, \leq)$, satisfying the following condition: for any pair $(\lambda, \mu) \in$ $\Lambda \times M$, there exists either and index $\lambda^{\prime} \in \Lambda$, with $\lambda^{\prime} \geq \lambda, \varphi\left(\lambda^{\prime}\right) \geq \mu$, or an index $\mu^{\prime} \in M$, with $\mu^{\prime} \geq \mu, \psi\left(\mu^{\prime}\right) \geq \lambda$. Compared to the condition from Theorem 2.1, here we do not assume that the functions $\varphi$ and $\psi$ are increasing. But, by [8] ( Ch.I, $\S 1.2$, Th.2), we can suppose that $(\Lambda, \leq)$ and $(M, \leq)$ are directed cofinite ordered sets, and then by [8] ( Ch.I, $\S 1.2$, Lemma 1), there exist increasing functions $\bar{\varphi}:(\Lambda, \leq) \rightarrow(M, \leq)$ and $\bar{\psi}:(M, \leq) \rightarrow(\Lambda, \leq)$ such that $\varphi \leq \bar{\varphi}$ and $\psi \leq \bar{\psi}$. Then $\bar{\varphi}\left(\lambda^{\prime}\right) \geq \varphi\left(\lambda^{\prime}\right) \geq \mu$ and $\bar{\psi}\left(\mu^{\prime}\right) \geq \psi\left(\mu^{\prime}\right) \geq \lambda$ respectively. Therefore $(\mathbf{X}, \mathbf{Y})$ satisfies the condition from Theorem 2.1 with respect to the pair of functions $(\bar{\varphi}, \bar{\psi})$. Thus, we see that the condition from Theorem 2.1 is somewhat less restrictive that it would seem.

\section{Some products in a shape category}

Let $S h_{(\mathcal{T}, \mathcal{P})}$ denote a shape category in the sense of Mardešić-Segal [8](Ch.I, $\S 2)$. The pair $(\mathcal{T}, \mathcal{P})$ consists of a category $\mathcal{T}$ and a dense subcategory $\mathcal{P}$ of $\mathcal{T}$ which means that every object $X$ of $\mathcal{T}$ has a $\mathcal{P}$-expansion, that is a promorphism $\mathbf{p}=\left(p_{\lambda}\right): X \rightarrow \mathbf{X}=\left(X_{\lambda}, p_{\lambda \lambda^{\prime}}, \Lambda\right)$ in $\mathcal{T}$, with $\mathbf{X}$ in pro $-\mathcal{P}$, so that the following condition is satisfied : for any morphism $\mathbf{h}: X \rightarrow \mathbf{Y}$ in pro $-\mathcal{T}$, with $\mathbf{Y}$ in pro $-\mathcal{P}$, there exists a unique morphism $\mathbf{f}: \mathbf{X} \rightarrow \mathbf{Y}$ in pro $-\mathcal{P}$ such that $\mathbf{h}=\mathbf{f} \circ \mathbf{p}$. Now the objects of the category $S h_{(\mathcal{T}, \mathcal{P})}$ are all the objects of $\mathcal{T}$, and if $X, Y$ are two objects of $\mathcal{T}$ with $\mathbf{p}: X \rightarrow \mathbf{X}$, $\mathbf{q}: Y \rightarrow \mathbf{Y}, \mathcal{P}$-expansions, then a shape morphism $F: X \rightarrow Y$ is a class of pro-morphisms $\mathbf{f}: \mathbf{X} \rightarrow \mathbf{Y}$ in pro $-\mathcal{P}$ with respect to the relation $\mathbf{f} \sim \mathbf{f}^{\prime}$ defined in the following way: if $\mathbf{p}^{\prime}: X \rightarrow \mathbf{X}^{\prime}, \mathbf{q}^{\prime}: Y \rightarrow \mathbf{Y}^{\prime}$ are $\mathcal{P}$-expansions, with some isomorphisms $\mathbf{i}: \mathbf{X} \rightarrow \mathbf{X}^{\prime}, \mathbf{j}: \mathbf{Y} \rightarrow \mathbf{Y}^{\prime}$, and $\mathbf{f}^{\prime}: \mathbf{X}^{\prime} \rightarrow \mathbf{Y}^{\prime}$, then we have $\mathbf{j} \circ \mathbf{f}=\mathbf{f}^{\prime} \circ \mathbf{i}$. Composition of shape morphisms $F: X \rightarrow Y, G: Y \rightarrow Z$ is defined by composing representatives $\mathbf{f}: \mathbf{X} \rightarrow \mathbf{Y}$ and $\mathbf{g}: \mathbf{Y} \rightarrow \mathbf{Z}$, and identity shape morphism $1_{X}: X \rightarrow X$ is defined by $1_{\mathbf{X}}: \mathbf{X} \rightarrow \mathbf{X}$. For every morphism $f: X \rightarrow Y$ in $\mathcal{T}$ and for $\mathcal{P}$-expansions $\mathbf{p}: X \rightarrow \mathbf{X}, \mathbf{q}: Y \rightarrow \mathbf{Y}$, there is a unique $\mathbf{f}: \mathbf{X} \rightarrow \mathbf{Y}$ in pro- $\mathcal{P}$ such that $\mathbf{f} \circ \mathbf{p}=\mathbf{q} \circ f$ in pro- $\mathcal{T}$. This correspondence is compatible with the relation $\sim$, so that we can associate with every $f \in \mathcal{T}(X, Y)$ a shape morphism $X \rightarrow Y$, i.e., the class of $f \in$ $($ pro $-\mathcal{P})(\mathbf{X}, \mathbf{Y})$, which is denoted by $S(f)$. If we put $S(X)=X$, we obtain a covariant functor $S: \mathcal{T} \rightarrow S h_{(\mathcal{T}, \mathcal{P})}$, the so- called the shape functor.

Let $S h_{(\mathcal{T}, \mathcal{P})}$ be a shape category with $\mathcal{T}$ and $\mathcal{P}$ categories with products, and $(X, Y)$ a pair of objects in category $\mathcal{T}$. Then in $\mathcal{T}$ there exists the product $X \times Y$ with the projections $\pi_{X}: X \times Y \rightarrow X$ and $\pi_{Y}: X \times Y \rightarrow Y$, and in pro $-\mathcal{P}$ there exists the product $\mathbf{X} \times \mathbf{Y}$ with the projections $\pi_{\mathbf{X}}: \mathbf{X} \times \mathbf{Y} \rightarrow$ 
$\mathbf{X}, \pi_{\mathbf{Y}}: \mathbf{X} \times \mathbf{Y} \rightarrow \mathbf{Y}$, for $\mathbf{p}: X \rightarrow \mathbf{X}$ and $\mathbf{q}: Y \rightarrow \mathbf{Y}, \mathcal{P}$-expansions of $X$ and $Y$ respectively (see Theorem 2.1, or more generally [2] and [3]). Then, because the subcategory $\mathcal{P}$ is dense in $\mathcal{T}$, there exists a $\mathcal{P}$-expansion $\mathbf{r}: X \times Y \rightarrow \mathbf{Z}_{X \times Y}$.

Now, the morphisms $\pi_{X}$ and $\pi_{Y}$ induce the pro-morphisms pro $-\pi_{X}$ : $\mathbf{Z}_{X \times Y} \rightarrow \mathbf{X}$ and pro $-\pi_{Y}: \mathbf{Z}_{X \times Y} \rightarrow \mathbf{Y}$ respectively, so that

$$
\mathbf{p} \circ \pi_{X}=\left(\text { pro }-\pi_{X}\right) \circ \mathbf{r}, \quad \mathbf{q} \circ \pi_{Y}=\left(\text { pro }-\pi_{Y}\right) \circ \mathbf{r} .
$$

Now, by the definition of the directed product in pro- $\mathcal{P}$, there exists a unique morphism

$$
\mathbf{s}: \mathbf{Z}_{X \times Y} \rightarrow \mathbf{X} \times \mathbf{Y}
$$

such that

$$
\pi_{\mathbf{X}} \circ \mathbf{s}=\text { pro }-\pi_{X}, \quad \pi_{\mathbf{Y}} \circ \mathbf{s}=\text { pro }-\pi_{Y},
$$

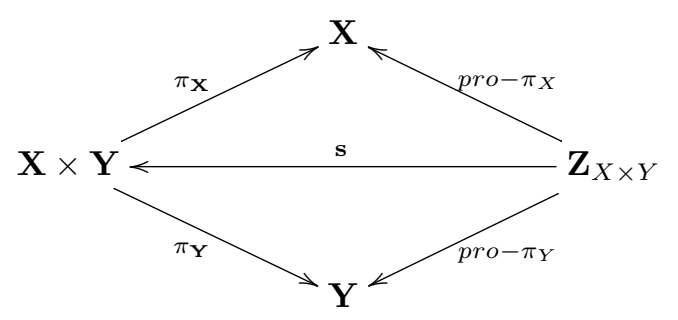

From relations (3.1) and (3.2) we obtain

$$
\pi_{\mathbf{X}} \circ(\mathbf{s} \circ \mathbf{r})=\mathbf{p} \circ \pi_{X}, \quad \pi_{\mathbf{Y}} \circ(\mathbf{s} \circ \mathbf{r})=\mathbf{q} \circ \pi_{Y} .
$$

Theorem 3.1. If the pro-morphism $\mathbf{s}: \mathbf{Z}_{X \times Y} \rightarrow \mathbf{X} \times \mathbf{Y}$ is an isomorphism, then the product $X \times Y$ in the category $\mathcal{T}$ is a product of the objects $X$ and $Y$ in the shape category $S h_{(\mathcal{T}, \mathcal{P})}$, with the projections $S h\left(\pi_{X}\right): X \times Y \rightarrow X$ and $\operatorname{Sh}\left(\pi_{Y}\right): X \times Y \rightarrow Y$.

Proof. Suppose that $Z$ is an arbitrary object in $S h_{(\mathcal{T}, \mathcal{P})}$ (i.e., $Z \in \mathcal{T}$ ), and $F_{X}: Z \rightarrow X, F_{Y}: Z \rightarrow Y$ are two shape morphisms. Let $\mathbf{t}: Z \rightarrow \mathbf{Z}$ be a $\mathcal{P}$-expansion of $Z$. Then the shape morphisms $F_{X}$ and $F_{Y}$ are given by some pro-morphisms $\mathbf{F}_{X}: \mathbf{Z} \rightarrow \mathbf{X}$ and $\mathbf{F}_{Y}: \mathbf{Z} \rightarrow \mathbf{Y}$, respectively, and these pro-morphisms induce a unique pro-morphism $\mathbf{F}: \mathbf{Z} \rightarrow \mathbf{X} \times \mathbf{Y}$ so that

$$
\pi_{\mathbf{X}} \circ \mathbf{F}=\mathbf{F}_{X}, \quad \pi_{\mathbf{Y}} \circ \mathbf{F}=F_{\mathbf{Y}},
$$




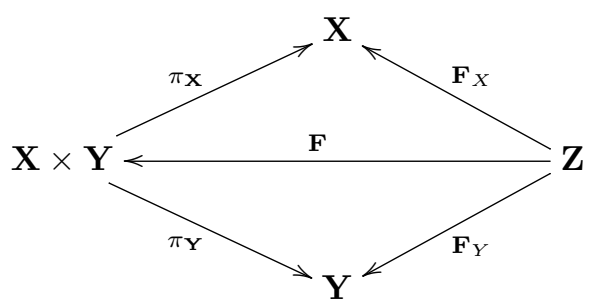

Now we compose the pro-morphism $\mathbf{F}$ and the pro-morphism $\mathbf{s}^{-1}: \mathbf{X} \times \mathbf{Y} \rightarrow$ $\mathbf{Z}_{X \times Y}$, which exists by hypothesis. Thus we obtain a pro-morphism

$$
\mathbf{s}^{-1} \circ \mathbf{F}: \mathbf{Z} \rightarrow \mathbf{Z}_{X \times Y}
$$

which defines a shape morphism

$$
F: Z \rightarrow X \times Y
$$

Finally, since we have the relations

$$
\begin{aligned}
& \left(\text { pro }-\pi_{X}\right) \circ \mathbf{s}^{-1} \circ \mathbf{F}=\mathbf{F}_{X}=\pi_{\mathbf{X}} \circ \mathbf{F}, \\
& \left(\text { pro }-\pi_{Y}\right) \circ \mathbf{s}^{-1} \circ \mathbf{F}=\mathbf{F}_{Y}=\pi_{\mathbf{Y}} \circ \mathbf{F},
\end{aligned}
$$

we deduce the reations

$$
S h\left(\pi_{X}\right) \circ F=F_{X}, \quad S h\left(\pi_{Y}\right) \circ F=F_{Y}
$$

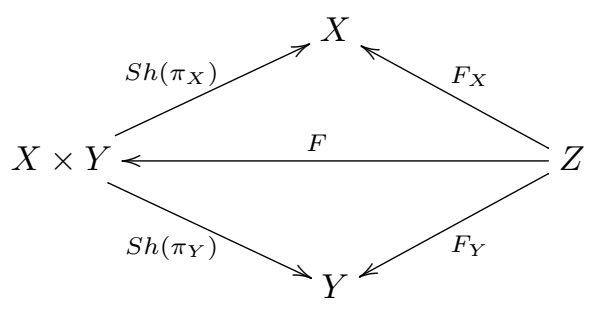

The uniqueness of $F$ results from the uniqueness of $\mathbf{F}$. This ends the proof.

Corollary 3.2. Suppose in a shape category $S h_{(\mathcal{T}, \mathcal{P})}$, with $\mathcal{T}$ and $\mathcal{P}$ categories with products, two $\mathcal{P}$-expansions $\mathbf{p}: X \rightarrow \mathbf{X}, q: Y \rightarrow \mathbf{Y}$ are given. Then if the pro-morphism $\mathbf{p} \times \mathbf{q}: X \times Y \rightarrow \mathbf{X} \times \mathbf{Y}$, satisfying $\pi_{\mathbf{X}} \circ(\mathbf{p} \times \mathbf{q})=$ $\mathbf{p} \circ \pi_{X}, \pi_{\mathbf{Y}} \circ(\mathbf{p} \times \mathbf{q})=\mathbf{q} \circ \pi_{Y}$, is a $\mathcal{P}$-expansion of the product $X \times Y$, then $\left(X \times Y, S h\left(\pi_{X}\right), S h\left(\pi_{Y}\right)\right)$ is a product in $S h_{(\mathcal{T}, \mathcal{P})}$ of the objects $X$ and $Y$. 
Corollary 3.3. Suppose given a shape category $S h_{(\mathcal{T}, \mathcal{P})}$ and in the category $\mathcal{T}$ two pairs $(X, Y)$ and $\left(X^{\prime}, Y^{\prime}\right)$ satisfying the condition from Theorem 3.1. If $f: X \rightarrow X^{\prime}$ and $g: Y \rightarrow Y^{\prime}$ are two morphisms in $T$, then the product $S h(f) \times S h(g)$ exists and it is equal to $S h(f \times g)$.

Proof. By Theorem 3.1, in the category $S h_{(\mathcal{T}, \mathcal{P})}$ there exist the products $(X \times$ $\left.Y, S h\left(\pi_{X}\right), S h\left(\pi_{Y}\right)\right)$ and $\left(X^{\prime} \times Y^{\prime}, S h\left(\pi_{X^{\prime}}\right), S h\left(\pi_{Y^{\prime}}\right)\right)$. Then the product of the shape morphisms $S h(f): X \rightarrow X^{\prime}, S h(g): Y \rightarrow Y^{\prime}$ exists and satisfies the relations

$$
\begin{aligned}
& S h\left(\pi_{X^{\prime}}\right) \circ(S h(f) \times S h(g))=S h(f) \circ S h\left(\pi_{X}\right), \\
& S h\left(\pi_{Y^{\prime}}\right) \circ(S h(f) \times S h(g))=S h(g) \circ S h\left(\pi_{Y}\right) .
\end{aligned}
$$

But by (2.1) we also obtain

$$
\begin{gathered}
S h\left(\pi_{X^{\prime}}\right) \circ S h(f \times g)=S h(f) \circ S h\left(\pi_{X}\right), \\
S h\left(\pi_{Y^{\prime}} \circ S h(f \times g)=S h(g) \circ S h\left(\pi_{Y}\right) .\right.
\end{gathered}
$$

Then the uniqueness of the product morphism implies the equality

$$
S h(f) \times S h(g)=S h(f \times g) .
$$

Using Theorem 3.1, we obtain a new proof of the following particular case of the Keesling' Theorem [4](Th.1.1).

Proposition 3.4. Consider the shape category $S h(T o p):=S h_{H T o p, H P o l}$ of topological spaces $([8]$, Ch.I,, ). Then the product in the category Sh(Top) of two metric compact spaces $X$ and $Y$ is the topological product $X \times Y$ with the shape projection $S h\left(\pi_{X}\right): X \times Y \rightarrow X$ and $\operatorname{Sh}\left(\pi_{Y}\right): X \times Y \rightarrow Y$.

Proof. First, we recall that for any two topological spaces $X$ and $Y$, with the product $\left(X \times Y, \pi_{X}, \pi_{Y}\right)$, we have that $\left(X \times Y, H\left(\pi_{X}\right), H\left(\pi_{Y}\right)\right)$ is the product of $X$ and $Y$ in HTop. Thus we can assume the hypothesis expressed in this form. Then it is known ([8], Ch.I, $\S 5.2$, Cor.4) that every compact metric space is the inverse limit of an inverse sequence of compact polyhedra. So, $X=\lim \mathbf{X}, Y=\lim \mathbf{Y}$, with $\mathbf{X}=\left(X_{n}, p_{n n+1}\right)$ and $\mathbf{Y}=\left(Y_{n}, q_{n n+1}\right)$. Moreover, if $\mathbf{p}: X \rightarrow \widehat{\mathbf{X} \text { and }} q: Y \rightarrow \mathbf{Y}$ are projections of inverse limit, then $\mathbf{H} p: X \rightarrow H \mathbf{X}$ and $H \mathbf{q}: Y \rightarrow H \mathbf{Y}$ are HPol -expansions ([8], Ch.I, $\S 5.3$, Th.9). Now we have $\mathbf{X} \times \mathbf{Y}=\left(X_{n} \times Y_{n}, p_{n n+1} \times q_{n n+1}\right), X \times Y=\lim (\mathbf{X} \times \mathbf{Y})$ , and if $\mathbf{r}: X \times Y \rightarrow \mathbf{X} \times \mathbf{Y}$ is the projection of inverse limit, $H \mathbf{r}: X \times Y \rightarrow$ $H(\mathbf{X} \times \mathbf{Y})$ is an HPol-expansion of $X \times Y$. By this we can identify the inverse sequences $\mathbf{X} \times \mathbf{Y}$ and $\mathbf{Z}_{X \times Y}$, and then the conclusion follows by Theorem 3.1 . 
Denote by $s d X$ the shape dimension of a topological space (see [8] Ch.II, $\S 1)$.

Corollary 3.5. If $X$ and $Y$ are two metric compact spaces with sd $X \leq m$ and $s d Y \leq n$, then $s d(X \times Y) \leq m+n$.

Proof. We use the notations from the proof of Proposition 3.4 and apply the following characterisation of shape dimension ([8], Ch.II, $\S 1.1$, Th.2): $s d X \leq n$ if and only if for an HPol-expansion $\mathbf{p}: X \rightarrow \mathbf{X}=\left(X_{\lambda}, p_{\lambda \lambda^{\prime}}, \Lambda\right)$ and every $\lambda \in \Lambda$, there is an index $\lambda^{\prime} \geq \lambda$ so that $p_{\lambda \lambda^{\prime}}$ factors in HPol through a polyhedron $P$ with $\operatorname{dim} P \leq n$. Then if $p_{\lambda \lambda^{\prime}}=v \circ u, u: X_{\lambda^{\prime}} \rightarrow P, v: P \rightarrow$ $X_{\lambda}, \operatorname{dim} P \leq m$, and $q_{\mu \mu^{\prime}}=v^{\prime} \circ u^{\prime}, u^{\prime}: Y_{\mu^{\prime}} \rightarrow Q, v^{\prime}: Q \rightarrow Y_{\mu}, \operatorname{dim} Q \leq n$, then $p_{\lambda \lambda^{\prime}} \times q_{\mu \mu^{\prime}}=(v \circ u) \times\left(v^{\prime} \circ u^{\prime}\right)=\left(v \times v^{\prime}\right) \circ\left(u \times u^{\prime}\right)$, and $\operatorname{dim}(P \times Q) \leq m+n$.

Proposition 3.6. Let $X$ and $Y$ be two metrizable or parcompact spaces such that there exist $X^{\prime}$ and $Y^{\prime}$ metric compact spaces satisfying $S h(X)=\operatorname{Sh}\left(X^{\prime}\right)$ and $S h(Y)=S h\left(Y^{\prime}\right)$. Then $\left(X \times Y, S h\left(\pi_{X}\right), S h\left(\pi_{Y}\right)\right)$ is a directed product of the objects $X$ and $Y$ in the category $S h(T o p)$.

Proof. If $\mathbf{p}: X \rightarrow \mathbf{X}, \mathbf{q}: Y \rightarrow \mathbf{Y}, \mathbf{p}^{\prime}: X^{\prime} \rightarrow \mathbf{X}^{\prime}, \mathbf{q}^{\prime}: Y^{\prime} \rightarrow \mathbf{Y}^{\prime}, \mathbf{r}: X \times Y \rightarrow$ $\mathbf{Z}_{X \times Y}, \mathbf{r}^{\prime}: X^{\prime} \times Y^{\prime} \rightarrow \mathbf{Z}_{X^{\prime} \times Y^{\prime}}$ are HPol-expansions, then in the pro-category pro-HPol, there exist some isomorphisms $\alpha: \mathbf{X} \rightarrow \mathbf{X}^{\prime}, \beta: \mathbf{Y} \rightarrow \mathbf{Y}^{\prime}$. But by [5](Th.3.1 and Th.3.5), $S h(X \times Y)=S h\left(X^{\prime} \times Y^{\prime}\right)$ holds, which implies the existence in pro-HPol of an isomorphism $\gamma: \mathbf{Z}_{X^{\prime} \times Y^{\prime}} \rightarrow \mathbf{Z}_{X \times Y}$, satisfying the relations $\pi_{\mathbf{X}^{\prime}} \circ \gamma=\alpha_{\mathbf{X}}$ and $\pi_{\mathbf{Y}^{\prime}} \circ \gamma=\beta \circ \pi_{\mathbf{Y}}$. Then with the notations from Theorem 3.1, we obtain

$$
(\alpha \circ \beta) \circ \mathbf{s}^{\prime}=\mathbf{s} \circ \gamma .
$$

Now by Proposition 3.4, the pro-morphism $\mathbf{s}^{\prime}: \mathbf{Z}_{X^{\prime} \times Y^{\prime}} \rightarrow \mathbf{X}^{\prime} \times \mathbf{Y}^{\prime}$ is an isomorphism. And since $\alpha \times \beta$ and $\gamma$ are also pro-isomomorphisms, we deduce that $\mathbf{s}: \mathbf{Z}_{X \times Y} \rightarrow \mathbf{X} \times \mathbf{Y}$ is itself an isomorphism. Then by Theorem 3.1, we conclude that $\left(X \times Y, S h\left(\pi_{X}\right), S h\left(\pi_{Y}\right)\right)$ is a product in $\operatorname{Sh}(\mathrm{Top})$ of the space $X$ and $Y$.

\section{Keesling and Dydak-Mardešić examples}

Keesling's Example [4]. For this example Keesling uses the Mardešić's generalization of Holsztyński's approach of the shape theory of topological spaces [1], [6].

Let $X$ be the set of points in the plane $R^{2}$ defined by 


$$
X=\left\{x=\left((1+t) \cos 2 \pi t^{-1},(1+t) \sin 2 \pi t^{-1}\right): 0<t \leq 1\right\} \cup\{(1,0)\} .
$$

The space $X$ consists of ray beginning at the point $z_{0}=(2,0)$ and spiraling to the circle $x^{2}+y^{2}=1$ (not included in $X$ ) and the point $z_{1}=(1,0)$ on the circle.

Suppose that $X \times X$ has in Top the projection $\pi_{1}$ and $\pi_{2}$, and that $X \times$ $X$ with $S h\left(\pi_{1}\right)$ and $S h\left(\pi_{2}\right)$ as projections is the product of $X$ with itself in $\mathrm{Sh}(\mathrm{Top})$. We prove that this is not true. For this purpose consider the constant maps $c_{0}, c_{1}: X \rightarrow X$, with $c_{0}(X)=(2,0), c_{1}(X)=(1,0)$. Since $X$ is connected we have $S h\left(c_{0}\right)=S h\left(c_{1}\right)$. Then we define the shape morphisms $F_{i}: X \rightarrow X$ by $F_{1}=S h\left(c_{0}\right)=\operatorname{Sh}\left(c_{1}\right)$ and $F_{2}=S h\left(i d_{X}\right)$. If $X \times X$ is the product in $\operatorname{Sh}$ (Top)with projections $S h\left(\pi_{1}\right), S h\left(\pi_{2}\right)$, then there is a unique shape morphism $F: X \rightarrow X \times X$, such that $\operatorname{Sh}\left(\pi_{i}\right) \circ F=F_{i}$ for $i=1,2$. But for $F$ we can take both $F^{\prime}=S h\left(c_{0} \times i d_{X}\right)$ and $F^{\prime \prime}=S h\left(c_{1} \times i d_{X}\right)$ , since $S h\left(\pi_{1}\right) \circ F^{\prime}=S h\left(\pi_{1} \circ\left(c_{0} \times i d_{X}\right)\right)=\operatorname{Sh}\left(c_{0}\right)=F_{1}, S h\left(\pi_{2}\right) \circ F^{\prime}=$ $S h\left(\pi_{2} \circ\left(c_{0} \times i d_{X}\right)\right)=S h\left(i d_{X}\right)=F_{2}, S h\left(\pi_{1}\right) \circ F^{\prime \prime}=S h\left(\pi_{1} \circ\left(c_{1} \times i d_{X}\right)\right)=$ $S h\left(c_{1}\right)=F_{1}, S h\left(\pi_{2}\right) \circ F^{\prime \prime}=S h\left(\pi_{2} \circ\left(c_{1} \times i d_{X}\right)\right)=F_{2}$. And we can prove that $F^{\prime} \neq F^{\prime \prime}$. For this we consider $X$ ordered by the linear order induced by the parameter $t$ and $(1,0) \leq x$ for all $x \in X$. Then we define the continuous map $g: X \times X \rightarrow X$, by $g(x, y)=\min \{x, y\}$ with this order. Now we have $S h(g) \circ F^{\prime}=S h(g) \circ S h\left(c_{0} \times i d_{X}\right)=S h\left(i d_{X}\right)$ and $S h(g) \circ F^{\prime \prime}=S h(g) \circ$ $S h\left(c_{1} \times i d_{X}\right)=S h\left(c_{1}\right)$. But we observe that $X$ does not have trivial shape since there is a map $f: X \rightarrow S^{1}$ which is not null homotopic. Therefore $S h(g) \circ F^{\prime} \neq S h(g) \circ F^{\prime \prime}$ which implies $F^{\prime} \neq F^{\prime \prime}$. We can conclude that $\left(X \times X, S h\left(\pi_{1}\right), S h\left(\pi_{2}\right)\right)$ it's not a product in $\operatorname{Sh}($ Top $)$.

Dydak -Mardešić example [10]. In [10] is proved that the Cartesian product $X \times P$ of the dyadic solenoid $X$ and the wedge $P=P_{1} \vee P_{2} \vee \ldots$ of a sequence of 1 -spheres is not a product in the shape category of topological spaces $\mathrm{Sh}$ (Top).

The dyadic solenoid $X$ is the limit of the inverse sequence $\mathbf{X}=\left(X_{n}, p_{n n+1}, \mathbb{N}\right)$ with $X_{n}=S^{1}=\left\{\zeta=e^{2 \pi i t} \mid 0 \leq t \leq 1\right\}$ and $p_{n n+1}(\zeta)=\zeta^{2}$, and with the canonical projection $p_{n}: X \rightarrow X_{n}$ the map $p_{n}\left(\zeta_{1}, \zeta_{2}, \ldots\right)=\zeta_{n}, n \in \mathbb{N}$.

The space $P$ is obtained from the coproduct $\amalg_{n=1}^{\infty} P_{n}, P_{n}=S^{1}$, by identifying all the base points $1 \in S^{1}$ in the various summands $P_{n}$ to a single base point $*$ of $P$.

Now for any fixed point $x \in X$ is defined $h^{x}: P \leftrightarrow X \times P$ by $h^{x}(t)=$ $(x, t), t \in P$. Then is proved that for an arbitrary choice of points $x, x^{\prime} \in X$, the mappings $h=h^{x}, h^{\prime}=h^{x^{\prime}}$ satisfy the conditions

$$
\operatorname{Sh}\left[\pi_{X}\right] \operatorname{Sh}[h]=S h\left[\pi_{X}\right] S h\left[h^{\prime}\right],
$$


(2) $\quad \operatorname{Sh}\left[\pi_{P}\right] \operatorname{Sh}[h]=S h\left[\pi_{P}\right] S h\left[h^{\prime}\right]$.

Finally it is proved that there exist points $x, x^{\prime} \in X$ such that $h=h^{x}, h^{\prime}=$ $h^{x^{\prime}}$ satisfy the condition

$$
S h[h] \neq S h\left[h^{\prime}\right] .
$$

The idea is the same as for Keesling's example but the proof is more complicated. Is defined a mapping $q: X \times P \rightarrow Q$, where $Q$ is a CW-complex obtained starting from $\mathbf{X}$ and $P$. For this map is provided that there exist points $x, x^{\prime} \in X$ such that $q h^{x} \neq q h^{x^{\prime}}$. Then, since $Q$ is a CW-complex, we obtain relation (1)-(3) for $h=h^{x}$ and $h^{\prime}=h^{x^{\prime}}$.

Based on these examples and using 3.1 and Continuity Theorem ([8], Ch.I, $\S 2.3$, Theorem 6 and Remark 13), we can state the following result:

Corollary 4.1. In the category $\operatorname{Sh}(\mathrm{Top})$ there exist inverse limits $X=\underline{\lim \mathbf{X}}$ , $Y=\underline{\lim } \mathbf{Y}$ such that

$$
X \times Y \nsucceq \underset{l i m}{\lim }(\mathbf{X} \times \mathbf{Y}) .
$$

\section{References}

[1] W. Holsztyǹski, An extension and axiomatic charcaterization of Borsuk's theory of shape, Fund. Math., 70 (1971), 1105-1108.

[2] I.C. Isaksen, A model structure for the category of pro-simplicial sets, Trans. Amer. Math. Soc. 353 (2001),2805-2841.

[3] I.C. Isaksen, Calculating limits and colimits in pro-categories, Fundam.Math. 175,No.2, 175-194 (2002).

[4] J. Keesling, Products in the shape category and some applications, Symp. Math. Inst. Nazionale di Alta Matematica 16 (1973), Academic Press, New York, 1974, 133-142.

[5] Y. Kodama, On shape of product spaces, General Topology and its Applications 8 (1978) 141-150.

[6] S. Mardešić, Shapes for topological spaces, Gen. Top. and its Appl., 3 (1973), 265-282.

[7] S. Mardešić, Products of compacta with polyhedra and topological spaces in the shape category, Mediterr. J. Math. 1 (2004), 43-49.

[8] S. Mardešić and J. Segal, Shape Theory. The Inverse System Approach, North Holand Publ. Co. 1982. 
[9] S. Mardešić and J. Segal, History of Shape Theory and its Application to General Topology, Handbook of the History of General Topology, Vol. 3, Kluwer Acad. Publ. 2001, pp. 1145-1179.

[10] J. Dydak and S. Mardešić, A counterexample concerning products in the shape category, Fundamenta Math. 186 (2005), pp. 39-54.

[11] D. Quillen, Homotopical Algebra, Lecture Notes in Math. 43, Springer Verlag (1967).

I. Pop

Faculty of Mathematics "Al.I.Cuza" University

700505-Iaşi, Romania

E-mail:ioanpop@uaic.ro 